\title{
Tableros con renovales de Nothofagus: efecto de la temperatura y el tiempo en el prensado
}

Particleboards from second growth wood of Nothofagus: Effect of pressing temperature and time

\author{
H. POBLETE ${ }^{1}$, J. SOTO ${ }^{2}, L$. INZUNZA ${ }^{1}$ \\ 1 Inst, de Tecnología de Productos Forestales, Universidad A ustral de Chile, Casilla 567, Valdivia, Chile. \\ 2 Ingeniería Ejec. Ind. Forestales, I. Prof. Diego Portales, Maipú 301, Concepción, Chile.
}

\section{SUMMARY}

The influence of temperature and pressing time on physical and mechanical properties of particleboard produced with a mixture of Nothofagus species was studied. Four temperatures $\left(130^{\circ} \mathrm{C}, 150^{\circ} \mathrm{C}, 170^{\circ} \mathrm{C}\right.$ and $\left.190^{\circ} \mathrm{C}\right)$ and three pressing times $\left(4,5\right.$ and $6 \mathrm{~min}$.) were applied. Board density was $700 \mathrm{~kg} / \mathrm{m}^{3}$.

The time needed to reach $100^{\circ} \mathrm{C}$ at the center of the board was reduced from 2 to 1 min. with higher pressing temperature. Bending strength was not affected by variables in the study. Internal bond was affected by press temperature and time. The effect of increasing press temperature was favorable to thickness swelling.

Key words: Particle boards, press time, press temperature, properties, Nothofagus.

\section{RESUMEN}

Se analiza la influencia de la temperatura y tiempo de prensado sobre las propiedades de tableros de partículas producidos con una mezcla de Nothofagus. Se aplicaron 4 temperaturas de prensado $\left(130^{\circ} \mathrm{C}, 150^{\circ} \mathrm{C}, 170^{\circ} \mathrm{C}, 190^{\circ} \mathrm{C}\right)$ y 3 tiempos de prensado (4, 5 y $6 \mathrm{~min}$.). Los tableros fueron elaborados con una densidad de $700 \mathrm{~kg} / \mathrm{m}^{3}$.

El tiempo para alcanzar $100^{\circ} \mathrm{C}$ en el centro del tablero fue reducido de 2 a 1 min., al aumentar la temperatura. La flexión no se vio afectada por las variables en estudio. La variación de la temperatura influyó directamente sobre la tracción. El hinchamiento se vio favorecido por el aumento de la temperatura de prensado.

Palabras claves: Tableros de partículas, tiempo prensado, temperatura prensado, propiedades, Nothofagus.

\section{INTRODUCCION}

La función del prensado con temperatura es la del polimerizar el adhesivo y densificar el material que compone el tablero. Durante el prensado se produce un proceso mecánico de deformación de las partículas por flexión y compresión, un calentamiento del material por la aplicación de temperatura y un proceso químico que conduce al fraguado de la resina sintética por policondensación. En este proceso interactúan una serie de factores: tipo de materia prima, humedad del material, tipo de adhesivo, presión, tiempo y temperatura de prensado, entre otros (Maloney, 1977).

La temperatura influye sobre la plastificación de las partículas y acelera la solidificación del adhesivo. En general, se necesita menor presión y tiempo para compactar el material a medida que aumenta la temperatura de los platos. Esto es resultado de un aceleramiento de la plastificación de la madera. Al variar la temperatura y el tiempo se obtiene, además, un cambio en las propiedades de los tableros (Liiri, 1969).

En el prensado el calor fluye desde los platos a través de la superficie del material hacia el interior. Esta transmisión de la temperatura provoca el fraguado del adhesivo, primero en la superficie y posteriormente en la zona central del tablero. La temperatura de los platos determina el tiempo que debe transcurrir para que en el centro del tablero se logre el fraguado del adhesivo.

En el caso del adhesivo Ureaformaldehído, la temperatura en el centro debe alcanzar a $100^{\circ} \mathrm{C}$ y 
el período de tiempo requerido para alcanzar los $100^{\circ} \mathrm{C}$ determina el tiempo total del ciclo de prensado (Kehr y Schoelze, 1965; Kelly, 1977). Poblete (1978) recomienda, para el cálculo del tiempo total de prensado en tableros con U reaformaldehído, uno a dos minutos más que el tiempo necesario para alcanzar $100^{\circ} \mathrm{C}$ en el centro del tablero.

Buschbeck y Kehr (1960) determinaron el tiempo para alcanzar $100^{\circ} \mathrm{C}$ en el centro del tablero, variando la temperatura entre $140^{\circ} \mathrm{C}$ y $200^{\circ} \mathrm{C}$ y el tiempo entre 2 y $16 \mathrm{~min}$. En este trabajo se Iograron $100^{\circ} \mathrm{C}$ con tiempos que variaron entre 0.8 y 3 $\min$.

Las propiedades físico-mecánicas se ven afectadas por los niveles de temperatura y tiempo que se empleen (Vital et al, 1974; Poblete, 1986; Buschbeck y Kehr, 1960).

Buschbeck y Kehr (1960) determinaron el hinchamiento en tableros con U reaformaldehído, prensados con temperaturas entre $110^{\circ} \mathrm{C}$ y $220^{\circ} \mathrm{C}$, y concluyen que con un tiempo de prensado corto y una temperatura alta se obtienen menores valores de hinchamiento. Resultados similares son reportados por Roffael et al. (1972) en tableros con Fenolformaldehído producidos con temperaturas entre $120^{\circ} \mathrm{C}$ y $220^{\circ} \mathrm{C}$.

Respecto a las propiedades mecánicas, Buschbeck y Kehr (1960) determinaron la influencia de la temperatura entre $140^{\circ} \mathrm{C}$ y $200^{\circ} \mathrm{C}$, con tiempos de 2 a 16 minutos. Estos autores registraron un mejoramiento de las resistencias con el aumento del tiempo de prensado de 2 a 7 minutos. Con tiempos de prensado más largos las propiedades disminuyeron.

Diversos autores han determinado que al aumentar la temperatura se incrementan las propiedades mecánicas (Roffael et al, 1972; Easton, 1986; M cCollum 1986). Heebink et al. (1972) aumentan la temperatura de prensado y comprueban que se produce una transferencia de calor más rápida, obteniéndose una mayor densidad en la parte central del tablero, lo que conduce a una mayor resistencia a la tracción y a una disminución de la flexión.

\section{MATERIAL Y METODOS}

2.1. material. Como material para la fabricación de los tableros de partículas se utilizó una mezcla de astillas producidas de árboles sin descortezar y sin ramas, de las especies roble
(Nothofagus obliqua), raulí (Nothofagus alpina) y un híbrido de ambas (Nothofagus sp.) provenientes del raleo de renovales ubicados en la VIII Región, en el área de protección Radal Siete Tazas. La participación de las especies en la mezcla fue de un tercio de cada una.

La selección de los árboles para el estudio se realizó considerando que los diámetros de las trozas debían fluctuar entre 10 y $20 \mathrm{~cm}$. Las características de la fabricación y preparación de las partículas se encuentra descrita en un trabajo anterior (Poblete, 1989).

2.2. FABRICACION DELOSTABLEROS. La fabricación de los tableros se realizó con las siguientes condiciones:

- Densidad de tablero : $700 \mathrm{~kg} / \mathrm{m}^{3}$

- Espesor del tablero: $11 \mathrm{~mm}$

- Condiciones de las partículas

$\begin{array}{ll}\text { Capa externa } & 40 \% \text { del material } \\ \text { Humedad capa externa } & 7 \% \\ \text { Capa media } & 60 \% \text { del material } \\ \text { Humedad capa media } & 4 \%\end{array}$

$\begin{array}{ll}\text { - Adhesivo } & \text { : U reaformaldehído } \\ \text { Adhesivo en capas } & \\ \text { externas } & : 9 \% \text { (solución } \\ & \text { al } 50 \%) \\ \begin{array}{l}\text { Adhesivo en capas } \\ \text { medias }\end{array} & : 7 \%(\text { solución } \\ & \text { al } 50 \%)\end{array}$

- Ciclo de prensado Temperaturas $130,150,170 \mathrm{y}$ $190^{\circ} \mathrm{C}$

Presión máxima $\quad 3.0 \mathrm{~N} / \mathrm{m} \mathrm{m}^{2}$ Presión media $\quad 1.5 \mathrm{~N} / \mathrm{m} \mathrm{m}^{2}$

Tiempos de prensado 4, 5 y $6 \mathrm{~min}$.

En el ciclo de prensado el tiempo a presión máxima se determinó agregando un minuto al tiempo necesario para alcanzar $100^{\circ} \mathrm{C}$ en el centro del tablero. El resto del tiempo se dividió en 30 segundos para bajar a la presión media, permaneciendo con $1.5 \mathrm{~N} / \mathrm{m} \mathrm{m}^{2}$ hasta el final del ciclo. Para lograr lo anterior, con una termocupla se midió la temperatura en el centro del tablero y se cronometró el tiempo necesario para alcanzar $100^{\circ} \mathrm{C}$.

2.3. PROPIEDAdes fisICO-MECANICAS. Las propiedades físico-mecánicas se determinaron mediante normas DIN. Las normas aplicadas fueron:

- Climatizado (DIN 50.014)

- Densidad y humedad (DIN 52.361)

- Resistencia a la flexión (DIN 52.362) 
- Hinchamiento (DIN 52.364)

- Resistencia a la tracción (DIN 52.365)

Los datos obtenidos de estos ensayos fueron evaluados comparándolos con las especificaciones de la norma DIN. El tratamiento estadístico comprendió un análisis de varianza, incluido test de Scheffe, considerando un nivel de confianza de un $95 \%$. Un análisis de la normalidad de los datos obtenidos fue realizado a través del test de Kolmogorow-Smirnov previo al análisis de varianza.

\section{PRESENTACION Y DISCUSION DE RESULTADOS}

3.1. efectos SOBREELPRENSADO. En la figura 1 se muestra cómo varía la temperatura en el centro del tablero, dependiendo de la temperatura de los platos $\left(190,170,150\right.$ y $\left.130^{\circ} \mathrm{C}\right)$ y del tiempo de prensado.

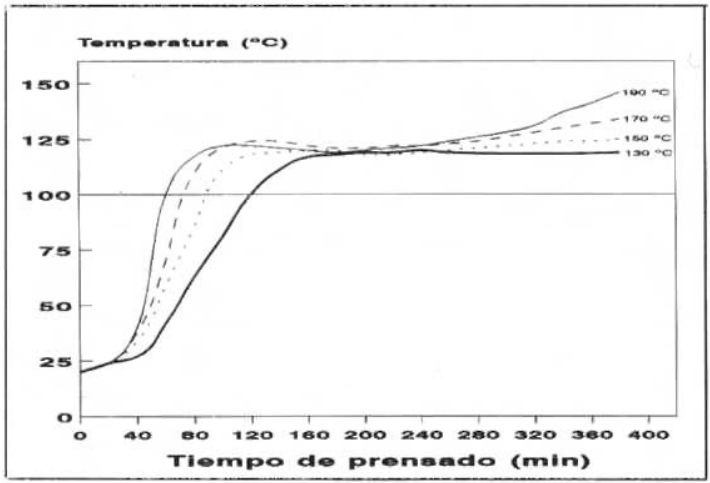

Figura 1. Efecto de la temperatura y tiempo de prensado sobre la temperatura en el centro del tablero.

Effect of pressing temperature and time on temperature at the center of the panel.

Se observa en la figura 1 un comportamiento similar en los diferentes ciclos de prensado. Al comienzo se produjo un ascenso lento de la temperatura dado por la transmisión del calor por conducción desde la capa externa hacia el interior. Posteriormente el ascenso de temperatura fue acelerado por la emigración del vapor generado en las capas externas hacia el centro. Este fenómeno se vio favorecido por la existencia de una gradiente de humedad entre las partículas de la capa externa (7\%) y la capa media (4\%).

En una tercera etapa se provocó un leve descenso de la temperatura y posterior esta- bilización, alcanzándose niveles similares ( 3 a 4 minutos de prensado) con una temperatura aproximada de $119^{\circ} \mathrm{C}$ (fig. 1). Lo anterior es producto de la liberación del vapor desde el centro del tablero hacia el exterior al bajar la presión de 3.0 a $1.5 \mathrm{~N} / \mathrm{mm}^{2}$. Por último, se produjo un ascenso de la temperatura una vez que es eliminado el vapor de agua (después de 4 minutos de prensado).

Las diferencias entre los ciclos de prensado se dieron en relación al tiempo en que se cumplieron las etapas descritas anteriormente. Los eventos ocurridos durante el ciclo de prensado para las distintas temperaturas aplicadas se presentan en el cuadro I.

\section{CUADRO I}

Efecto de la temperatura sobre el ciclo de prensado.

Effect of temperature on pressing time.

\begin{tabular}{|c|c|c|c|c|c|c|}
\hline \multirow{2}{*}{$\begin{array}{c}\text { Tempe } \\
\text { ratura } \\
\text { de pren- } \\
\text { sado }\end{array}$} & \multirow{2}{*}{$\begin{array}{l}\text { Cierre } \\
\text { de } \\
\text { prensa } \\
\text { min. }\end{array}$} & \multirow{2}{*}{$\begin{array}{c}\text { Tiempo } \\
\text { para } \\
100^{\circ} \mathrm{C} \\
\text { en centro } \\
\text { min. }\end{array}$} & \multirow{2}{*}{$\begin{array}{l}\text { Tiempo } \\
\text { A } \\
\text { Pmáx } \\
\text { min. }\end{array}$} & \multicolumn{3}{|c|}{$\begin{array}{c}\text { Tiempo A } \\
\text { Pmedia POR CICLO }\end{array}$} \\
\hline & & & & $4 \min$ & $5 \mathrm{~min}$. & $6 \mathrm{~min}$. \\
\hline $130^{\circ} \mathrm{C}$ & 1.80 & 2.0 & 3.0 & 1.0 & 2.0 & 3.0 \\
\hline $150^{\circ} \mathrm{C}$ & 1.50 & 1.5 & 2.5 & 1.5 & 2.5 & 3.5 \\
\hline $170^{\circ} \mathrm{C}$ & 1.15 & 1.3 & 2.3 & 1.7 & 2.7 & 3.7 \\
\hline $190^{\circ} \mathrm{C}$ & 0.85 & 1.0 & 2.0 & 2.0 & 3.0 & 4.0 \\
\hline
\end{tabular}

Al aumentar la temperatura de prensado las partículas se plastificaron en forma más rápida, por lo que el cierre de los platos se produjo más rápidamente y el calor se transmitió aceleradamente al centro del tablero, alcanzando los $100^{\circ} \mathrm{C}$ en el centro en un tiempo menor (cuadro 1). Observaciones similares son reportadas por Buschbeck y Kehr (1960) y Maloney (1977).

En general, para alcanzar $100^{\circ} \mathrm{C}$ en el centro del tablero se necesitó entre 1 y 2 minutos dependiendo de la temperatura aplicada. En todos los casos la presión máxima se mantuvo 60 segundos después de alcanzar los $100^{\circ} \mathrm{C}$ en el centro del tablero, lo que dio origen a diferentes ciclos de prensado, tal como se presentan en el cuadro 1. 
3.2.1. Hinchamiento 2 horas en agua. Los resultados de hinchamiento con 2 horas de inmersión en agua a $20^{\circ} \mathrm{C}$ se presentan en la figura 2 .

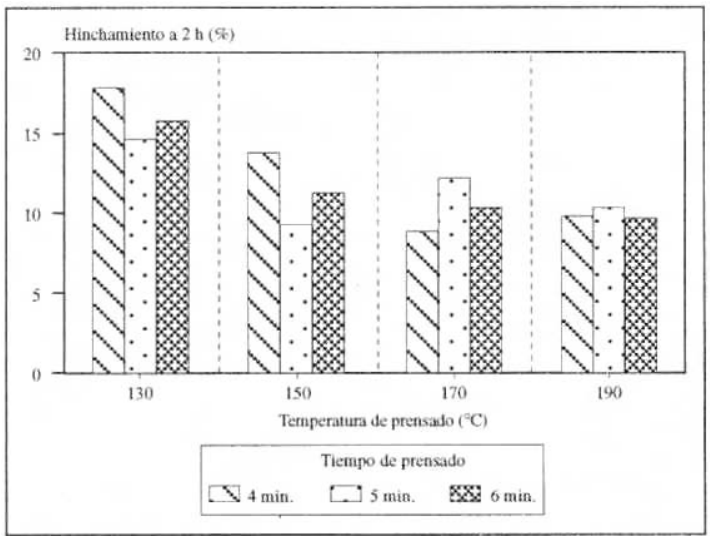

Figura 2. Hinchamiento a 2 horas inmersión en agua a $20^{\circ} \mathrm{C}$.

Thickness swelling after 2 hours in water at $20^{\circ} \mathrm{C}$.

Debe destacar que los mejores resultados de hinchamiento se obtuvieron al aumentar la temperatura de prensado. Sin embargo, los resultados obtenidos no cumplen con la exigencia de la norma DIN 68763, máximo $=8 \%$. Por lo anterior, Ios tableros deberían ser mejorados con la adición de un agente hidrófobo.

Los valores de hinchamiento obtenidos son semejantes a los mejores hinchamientos obtenidos por Poblete (1989) con una muestra de madera de la misma zona. En el trabajo citado se fabricaron tableros con las especies de Nothofagus por separado, obteniéndose hinchamientos entre $10 \%$ y $16 \%$.

El análisis de significancia del ensayo de hinchamiento a las dos horas en agua, para las variables temperatura y tiempo de prensado, se muestra en el cuadro 2.

$L$ as diferencias entre el hinchamiento logrado con las temperaturas más altas $\left(190^{\circ} \mathrm{C}\right.$ y $\left.170^{\circ} \mathrm{C}\right)$ y las más bajas $\left(150^{\circ} \mathrm{C}\right.$ y $130^{\circ} \mathrm{C}$ ) son significativas (cuadro 2).

El análisis de varianza de las variables indica que el tiempo de prensado no afecta directamente al hinchamiento, siendo la temperatura aplicada durante el prensado el factor más importante. Buschbeck y Kehr (1960) determinan un comportamiento similar.
A nálisis estadístico del hinchamiento a 2 horas. Statistical analysis of thickness swelling after 2 hours.

\begin{tabular}{|cccccc|}
\hline $\begin{array}{c}\text { Tempe- } \\
\text { ratura } \\
\left({ }^{\circ} \mathrm{C}\right)\end{array}$ & $\begin{array}{c}\text { Hinch. } \\
(\%)\end{array}$ & $\begin{array}{c}\text { Dif. } \\
\text { Sig. }\end{array}$ & $\begin{array}{c}\text { Prensado } \\
\text { min. }\end{array}$ & $\begin{array}{c}\text { Hinch. } \\
(\%)\end{array}$ & $\begin{array}{c}\text { Dif. } \\
\text { Sig. }\end{array}$ \\
\hline 190 & 10.28 & $\mathbf{3}$ & 5 & 11.71 & $\mathbf{3}$ \\
170 & 10.72 & $\mathbf{3}$ & 6 & 12.04 \\
150 & 11.47 & & 4 & 12.62 & $\mathbf{3}$ \\
130 & 16.10 & & & & \\
\hline
\end{tabular}

No presentan diferencias significativas

3.2.2. Hinchamiento a 24 horas. En la figura 3 se presentan los resultados de hinchamiento a 24 horas de inmersión en agua a $20^{\circ} \mathrm{C}$.

La norma DIN 68763 considera un límite de hinchamiento de $16 \%$ para este ensayo, nivel que no es superado por los tableros. Como se señalara, se estima que al agregar un hidrófobo, emulsión de parafina, podría cumplirse con el requisito de la norma.

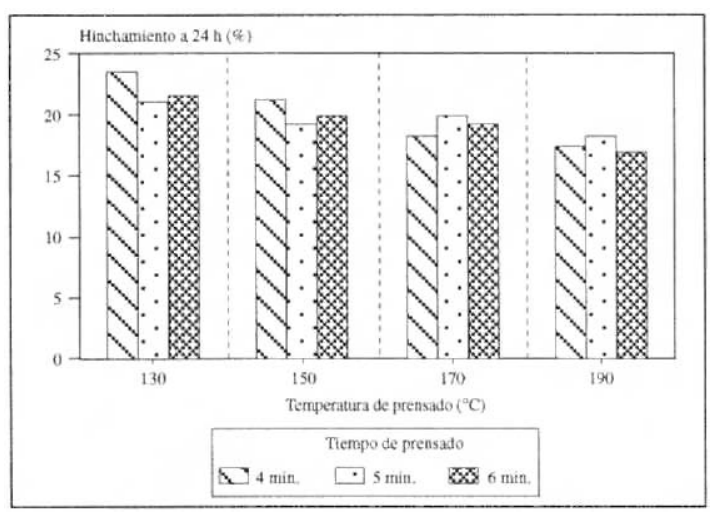

Figura 3. Hinchamiento a 24 horas inmersión en agua a $20^{\circ} \mathrm{C}$.

Thickness swelling after 24 hours in water at $20^{\circ} \mathrm{C}$.

El ensayo con una inmersión de 24 horas resulta importante, ya que provoca un hinchamiento que alcanza su grado máximo, permitiendo observar con mayor precisión las diferencias existentes entre los tratamientos. Es así como se pudo determinar que los mejores resultados se obtuvieron al aplicar altas temperaturas de prensado. Lo anterior coincide con los resultados obtenidos por otros investigadores (Buschbeck y Kehr, 1960; Roffael et al., 1972). 
Poblete (1989) trabajando con madera de $N$. obliqua, N. alpina y un híbrido de ambos, obtuvo valores de hinchamiento a las 24 horas semejantes a los del presente estudio.

El análisis de varianza se presenta en el cuadro 3 , y demuestra que la variable tiempo de prensado no tiene una incidencia importante sobre la propiedad, provocando un leve mejoramiento del hinchamiento al aumentar el tiempo de prensado. La temperatura de prensado como variable independiente afectó en forma inversa a los resultados de hinchamiento, siendo mejor la aplicación de una alta temperatura de prensado, como lo demuestra el cuadro 3.

\section{CUADRO 3}

A nálisis estadístico del hinchamiento a 24 horas. Statistical analysis of thickness swelling after 24 hours.

\begin{tabular}{|cccccc|}
\hline $\begin{array}{c}\text { Tempe- } \\
\text { ratura } \\
\left({ }^{\circ} \mathrm{C}\right)\end{array}$ & $\begin{array}{c}\text { Hinch. } \\
(\%)\end{array}$ & $\begin{array}{c}\text { Dif. } \\
\text { Sig. Prensado Hinch. } \\
\text { min. }\end{array}$ & $\begin{array}{c}\text { Dif. } \\
(\%)\end{array}$ \\
\hline 190 & 17.84 & 4 & 20.10 & \\
170 & 19.27 & 5 & 19.71 & \\
150 & 20.11 & 6 & 19.54 & \\
130 & 21.98 & & & & \\
\hline
\end{tabular}

Wo presentan diferencias significativas

\subsection{PROPIEDADES MECANICAS}

3.3.1. Resistencia a la flexión. Los análisis estadísticos indicaron que no existen diferencias significativas entre los tableros por efecto de la temperatura o del tiempo de prensado (cuadro 4).

Es necesario tener presente que la resistencia a la flexión está determinada por la calidad de las capas externas del tablero, las que en todos los casos estuvieron con temperatura y tiempo de prensado suficientes para obtener un fraguado adecuado del adhesivo. Por lo anterior resulta normal que no existan diferencias significativas entre los tratamientos, ya que las variaciones de la resistencia no son atribuibles a la calidad del fraguado del adhesivo.

$L$ as flexiones ( $M O R$ ) registradas son similares a las obtenidas por Poblete (1989) con $N$. alpina y superiores a las obtenidas por el mismo autor con $N$. obliqua y un híbrido de ambas especies.

\section{CUADRO 4}

A nálisis estadístico de resistencia a la flexión Statistical analysis of bending strength.

\begin{tabular}{|cccccc|}
\hline $\begin{array}{c}\text { Tempe- } \\
\text { ratura } \\
\left({ }^{\circ} \mathrm{C}\right)\end{array}$ & $\begin{array}{c}\text { Hinch. } \\
(\%)\end{array}$ & $\begin{array}{c}\text { Dif. } \\
\text { Sig. }\end{array}$ & $\begin{array}{c}\text { Prensado } \\
\text { min. }\end{array}$ & $\begin{array}{c}\text { Hinch. } \\
(\%)\end{array}$ & Sig. \\
\hline 130 & 18.72 & 4 & 18.85 & \\
150 & 18.69 & 5 & 18.59 & \\
170 & 18.52 & 6 & 18.34 & \\
190 & 18.46 & & & & \\
\hline
\end{tabular}

Wo presentan diferencias significativas

Cabe destacar que la flexión de los tableros es similar al límite exigido por la norma DIN (18 N/ $\mathrm{mm}^{2}$ ). Debido a que la forma de las partículas afecta la resistencia de los tableros, es posible aseverar que un cambio de la geometría de éstas, durante el viruteado, podría aumentar la flexión.

3.3.2. Resistencia a la tracción. Los resultados del ensayo se presentan en la figura 4. Los valores obtenidos demuestran que a medida que aumenta la temperatura de los platos de la prensa se obtienen mejores resistencias a la tracción. El notorio efecto de la temperatura se debe a que esta resistencia está dada por la adhesión obtenida en el centro del tablero. Por consiguiente, un aumento de temperatura de prensado implicó un mayor tiempo a temperatura superior a $100^{\circ} \mathrm{C}$ en el centro del tablero. De esta forma se influenció el fraguado del adhesivo, originándose una mayor resistencia de la unión adhesivo-madera y un aumento de la rigidez alcanzada por el adhesivo.

Los resultados obtenidos por los tableros fueron superiores a las exigencias mínimas estipuladas por la norma DIN $68763\left(0.4 \mathrm{~N} / \mathrm{mm}^{2}\right)$. LoS valores de tracción más altos se registraron al aplicar la temperatura más alta $\left(190^{\circ} \mathrm{C}\right)$ y el tiempo de prensado más corto ( 4 minutos). Resultados simiIares obtuvieron Buschbeck y Kehr (1960) aplicando altas temperaturas y tiempos de prensado cortos.

Los resultados obtenidos son similares a los registrados por Poblete (1989), quien al aplicar $160^{\circ} \mathrm{C}$ y 5 minutos logró $1.0 \mathrm{~N} / \mathrm{mm}^{2}$ con $N$. obliqua, $0.7 \mathrm{~N} / \mathrm{mm}^{2}$ con $N$. alpina y $1.0 \mathrm{~N} / \mathrm{m} \mathrm{m}^{2}$ con un híbrido de ambos.

Los resultados de los análisis estadísticos se presentan en el cuadro 5. 


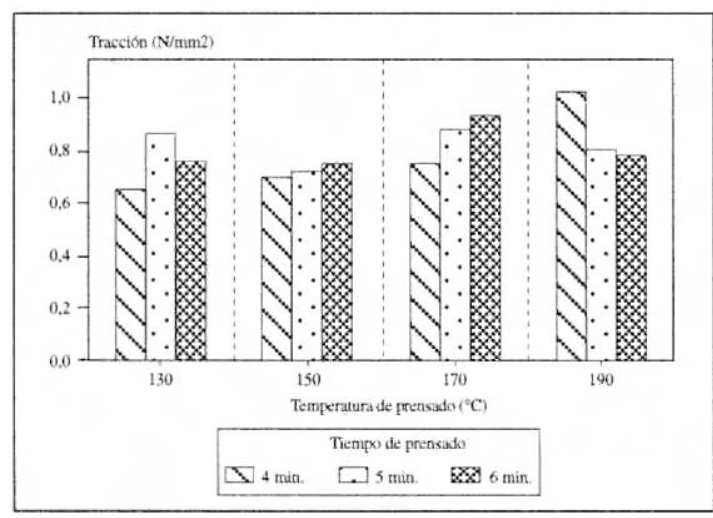

Figura 4. Resistencia a la tracción. Internal bond.

\section{CUADRO 5}

Resistencia a la tracción: significancia de las variables temperatura y tiempo de prensado.

Resistance to traction: significance of temperature and pressing time variables.

\begin{tabular}{|cccccc|}
\hline $\begin{array}{c}\text { Tempe- } \\
\text { ratura } \\
\left({ }^{\circ} \mathrm{C}\right)\end{array}$ & $\begin{array}{c}\text { Tracción } \\
(\%)\end{array}$ & $\begin{array}{c}\text { Sif. } \\
\text { Sig. }\end{array}$ & $\begin{array}{c}\text { Prensado Tracción } \\
\text { min. }\end{array}$ & $\begin{array}{c}\text { Dif. } \\
(\%)\end{array}$ & Sig. \\
\hline 130 & 0.711 & & 4 & 0.774 & \\
150 & 0.757 & & 5 & 0.792 & \\
170 & 0.839 & 6 & 0.805 & \\
190 & 0.855 & & & & \\
\hline
\end{tabular}

X. No presentan diferencias significativas

La variación de la temperatura provocó cambios significativos en la resistencia, lo que no ocurre con el tiempo de prensado. Pese a lo anterior se observó un leve incremento de esta propiedad al aumentar el tiempo del ciclo de prensado.

\section{CONCLUSIONES}

La temperatura de prensado influyó en forma directa sobre el tiempo necesario para alcanzar $100^{\circ} \mathrm{C}$ en el centro del tablero, disminuyendo el tiempo requerido al aumentar la temperatura de los platos. La aplicación de altas temperaturas permite la aplicación de un tiempo de prensado más corto.

El análisis del tiempo de prensado, como variable aislada, indicó no presentar una significancia estadística sobre las propiedades físicas y mecánicas de los tableros.
La propiedad física hinchamiento fue más afectada por la temperatura que por el tiempo total del ciclo de prensado. El aumento de la temperatura provocó disminuciones del hinchamiento. Los tableros no alcanzaron los mínimos exigidos por la norma, esta deficiencia puede ser corregida al incluir un hidrófobo.

La propiedad mecánica de flexión registró valores cercanos al límite establecido por la norma (18 $\left.\mathrm{N} / \mathrm{mm}^{2}\right)$. Los valores pueden ser mejorados cambiando la forma de las partículas durante el viruteado. En esta propiedad no se vio un efecto de la temperatura y tiempo de prensado.

La tracción perpendicular se vio favorecida a medida que aumentó la temperatura de prensado. Todos los tableros alcanzaron valores de tracción superiores a los exigidos por la norma DIN.

Debido a que el tiempo de prensado no afectó en forma directa a las propiedades ensayadas, se puede concluir que bastaría con un ciclo de sólo cuatro minutos para obtener resultados satisfactorios. Esto es de gran importancia desde el punto de vista económico, ya que posibilitaría un aumento de la producción.

\section{5. $B|B L I O G R A F| A$}

BUSCHBECK, L., E. KEHR. 1960. "Untersuchungen zur Verkurzung der Presszeit beim Heisspressen von Spanplatten", Holztechnologie 1(2): 112-123.

EASTON, J. 1986. Comportamiento de las propiedades físicomecánicas de un tablero compuesto al variar algunos factores de fabricación. Tesis, Universidad Austral de Chile, Facultad de Ciencias Forestales, $38 \mathrm{pp}$.

HEEBINK, B., W. LEHMANN, F. HEFTY. 1972. Reducing particleboard pressing time: Exploratory study. USDA For. Serv. Res. Pap. FPL 180. For. Prod. Lab. Madison, Wis.

KEHR, E., S. SCHOELZE. 1965. "The investigation on pressing conditions in the manufacture of particleboard", Drev. Vysk. 1965 (3): 133-147.

KELLY, M. 1977. Critical Literature Review of Relationships between processing Parameters and Physical Properties of Particleboard. USDA For. Serv. Gen. Tech. Rep., FPL-10, $66 \mathrm{pp}$.

LIIRI, O. 1969. "The pressure in the particleboard production", Holz als Roh-und Werkstoff 27(10): 371-378.

MALONEY, T. 1977. Modern particleboard and dry-process fiberboard manufacturing. Miller Freanan, San Francisco, $672 \mathrm{pp}$.

MC COLLUN, M. 1986. "Predicting modulus of rupture for hardboard siding substrate using hot-press operating conditions", For. Prod. J. 36(5): 36-38.

MOSLEMI, M. 1974. Particleboard. Vol. 2: Technology. Southerm III. Univ. Press Illinois, 245 pp.

PEREDO, M., H. POBLETE, L. NAVEILLAN. 1993. "Utilización de renovales de roble y raulí en la fabricación de tableros de partículas", Ciencia e Investigación Forestal 7(2): 215-239.

POBLETE, H. 1978. Uniones de madera con adhesivos. Publi- 
catión Técnica No 1 , Universidad Austral de Chile, Facultad de Ciencias Forestales, Valdivia, $43 \mathrm{pp}$.

POBLETE, H. 1986. "Resistencia mecánica de tableros de partículas producidos con mezclas de especies chilenas", Bosque 7(1): 38-45.

POBLETE, H. 1989. "Tableros de partículas con renovales de roble (Nothofagus obliqua), raulí (N. alpina) y un híbrido de ambos", Bosque 10(1): 9-17.

POBLETE, H., M. PERED O. 1990. "Tableros de desechos del debobinado de especies chilenas", Bosque 11(2): 45-58.

POBLETE, H. 1992. "Einfluss der Holzart und der
Holzartenmischung auf die mechanischen Eigenschaften von Spanplatten bei V erwendung chilenischer Hölzer", Holz als Roh- und Werkstoff 50(1992): 392-394.

ROFFAEL, E., K. SCHALLER, W. RAUCH. 1972. "Zur Fertigung von Phenolharzspanplatten", Holz-Zentralblatt. 93(136): 2003-2004.

VITAL, B., N. LEHMANN, R. BOONE. 1974. "How species and boad densities affect properties of exotic hardwood particleboards", For. Prod. J. 24(12): 37-45. 